Zagazig Veterinary Journal

Volume 44, Number 1, p. 21-28, June, 2016

CFaculty of Veterinary Medicine, Zagazig University, 44511, Egypt

DOI: 10.21608/zvjz.2016.7828.

\title{
Radiographic Examination of Rabbit Foeti Under the Effect of Hypervitaminosis A
}

Ahmed M.E. Omar, El-Sayed M.M. Basha, Salah El-dein A. Ahmed and Mohamed A. Aref* Anatomy and Embryology Department, Faculty of Veterinary Medicine, Zagazig University, 44511, Egypt

\section{Abstract \\ Article History: Received: 24/2/2016 Received in revised form: 8/6/2016 Accepted: 27/6/2016}

Fifteen full term rabbit foeti representing control and four treated groups (three from each group) were inspected by X-rays device at dorsoventral, ventrodorsal and bilateral views to assess the normal developmental process of the skeleton (control group) and skeletal malformations after excess dosage of vitamin A (treated groups). There were ossification centers represented by two radiolucent lines extending along the vertebral column as well as two radiolucent spots representing the ossification centers in the pelvis in all groups. The secondary ossification centers of the extremities were radiographically investigated in the proximal and distal extremities of humerus, femur and proximal extremity of tibia. Incomplete fusion, leaving an epiphyseal line, was found in the proximal and distal epiphyses of long bones. The results of our study provide a schematic atlas of normal examinations of the rabbit skeleton and its ossification centers. Such findings are useful in the interpretation of any cross- sectional pictures in this species and elucidate the impact of hypervitaminosis A on skeletal development.

Keywords: Radiography, Rabbits, Hypervitaminosis A

\section{Introduction}

Rabbits are considered a good source for meat and fur production, besides being ideal animals for laboratory researches. The normal development of rabbits requires a careful balance of retinoid concentrations [1]. High retinoid concentrations result in risk of fetal anomalies. Radiography (X-ray) provides an alternative imaging technology for the visualization of fetal skeletal specimens [2].

The X-beam technique creates just a twodimensional projection, to empower the whole skeleton to be visualized with sufficient detail to allow examination [3,4]. It has been used widely to study in vivo small animal creature models and in vitro specimens at relatively high resolutions $[5,6]$. The technique is now utilized for the visualization of fetal skeleton rather than old alizarin red staining technique particularly for the examination of ossification centers [7$10]$.
The lack of easy and non-hazardous imaging contrast agent protocols precludes their use in routine developmental and reproductive studies [11,12]. The development of the secondary ossification centers of the epiphyses was radiographically investigated in rabbits [13]. The ossification centers appeared at full term in the proximal and distal epiphyses of humerus, the head of femur, the distal epiphysis of femur and the proximal epiphysis of tibia.

Vitamin A is essential for the regeneration of the eye, normal skeletal development and maintenance of normal epithelial tissues [14]. However, hypervitaminosis A has been associated with alterations in mineral metabolism resulting in osteopenia, fractures, deformities and growth arrest [15]. The current study aimed to observe the rabbit skeleton and investigate its ossification centers by X-ray under the effect of hypervitaminosis $\mathrm{A}$ in full term foeti.

*Corresponding author e-mail: (abdelazizanatomy11@gmail.com), Anatomy and Embryology Department, Faculty of Veterinary Medicine, Zagazig University, 44511, Egypt. 


\section{Material and Methods}

\section{Animal groups}

This work was carried out on thirty rabbits comprising of twenty-five pregnant females weighting $2.5-3 \mathrm{~kg}$ (5-6 months old) and five male rabbits weighting 3-3.5 kg (3-4 months old) for mating. The twenty-five pregnant female rabbits were then divided into five equal groups, five rabbits in each group. The first control group did not receive any treatment. The animals in the second group were injected subcutaneously with vitamin A in a dose of 100 $\mathrm{mg} / \mathrm{kg} \mathrm{BW}$ per day from $3^{\text {rd }}$ to $6^{\text {th }}$ days of pregnancy [16-18]. While rabbits in the third, fourth and fifth groups were injected with the same dose at $7^{\text {th }}$ to $10^{\text {th }}$ days, $11^{\text {th }}$ to $14^{\text {th }}$ days and $15^{\text {th }}$ to $19^{\text {th }}$ days of pregnancy, respectively. After all pregnant rabbits gave birth of full term foeti, fifteen foeti (three from each group) were radiographed for skeletal examination.

\section{Radiographic examination}

Dorsoventral, ventrodorsal and bilateral views were radiographed using a mobile Fischer X-ray machine (Toshiba Rotanode, H.G. Fischer, inc. Franklin Park, Illinois, USA) at Surgery, Anesthesia and Radiology Department, Faculty of Veterinary Medicine, Zagazig University, with maximum output of $40-65 \mathrm{kV}$ and 3.2 to $6.3 \mathrm{~mA}$ and POX-300 BT.
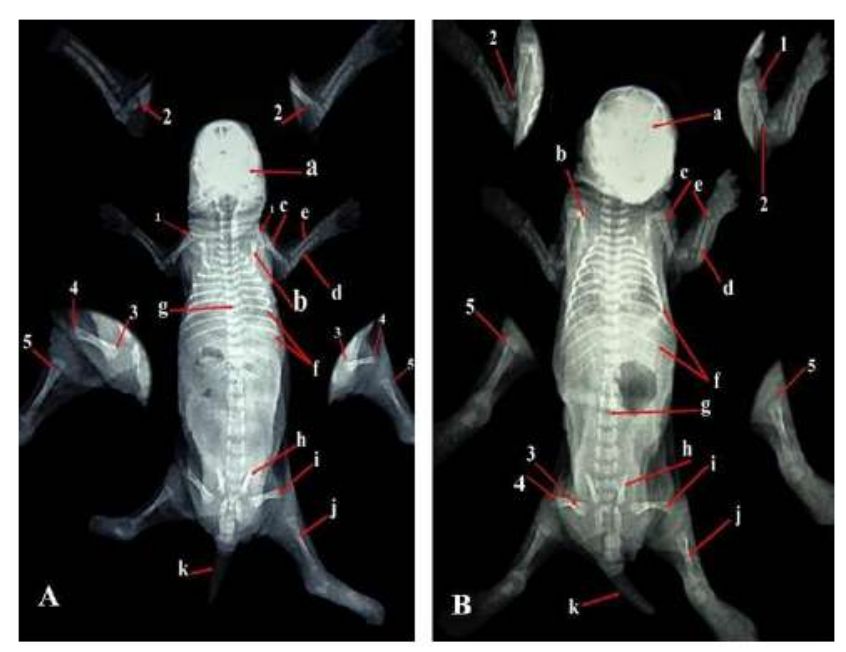

Plate 1 (A): A radiographic image of rabbit skeleton (first control group) (dorsoventral view) showing, skull (a), scapula (b), humerus (c), ulna (d), radius (e), ribs (f), vertebral column (g), coxal bone (h), femur (i), tibia (j) and caudal vertebrae (tail) (k), normal ossification center at proximal extremity of humerus (1), normal ossification center at distal extremity of humerus (2), normal ossification center at proximal extremity of femur (3), normal ossification center at distal extremity of femur (4) and normal ossification center at proximal extremity of tibia (5). Plate 1 (B): A radiographic image of rabbit skeleton (first control group) (ventrodorsal view) showing, skull (a), scapula (b), humerus (c), ulna (d), radius (e), ribs (f), vertebral column (g), coxal bone (h), femur (i), tibia (j) and caudal vertebrae (tail) (k), normal ossification center at proximal extremity of humerus (1), normal ossification center at distal extremity of humerus (2), normal ossification center at proximal extremity of femur (3), normal ossification center at distal extremity of femur (4) and normal ossification center at proximal extremity of tibia (5).

The radiographic image of rabbit skeleton of the second group showed future bony structures of scapula, humerus (absence of ossification center at proximal extremity of humerus, normal ossification center at distal extremity of humerus), radius and ulna. In the hind appendicular skeleton, future bony structures of coxal bone, femur (absence of 
ossification center at proximal and distal extremities of femur) and tibia (normal ossification center at proximal extremity of tibia) were clear (Plate. 1D,2A and 2B).

In the third group, in the fore appendicular skeleton, future bony structures of scapula, humerus (absence of ossification center at proximal extremity of humerus, normal ossification center at the distal extremity of humerus), radius and ulna were detected. In the hind appendicular skeleton, detected future bony structures of coxal bone, femur (absence of ossification center at proximal and distal extremities of femur), tibia (normal ossification center at proximal extremity of tibia) (Plate. $2 \mathrm{C}, 2 \mathrm{D}$ and $3 \mathrm{~A})$.
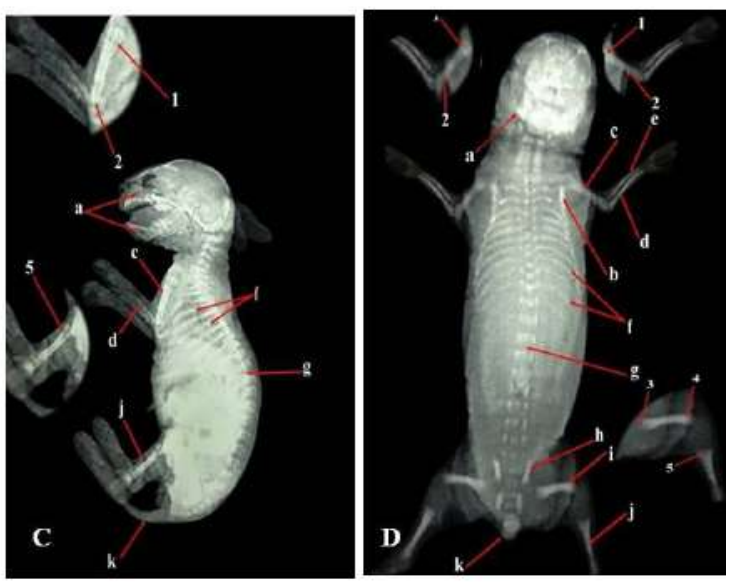

The fourth group, in the fore appendicular skeleton, future bony structures of scapula, humerus (normal ossification center at proximal extremity of humerus, absence of ossification center at distal extremity of humerus), radius and ulna were observed. In the hind appendicular skeleton, future bony structures of coxal bone, femur (absence of ossification center at proximal and normal ossification center at distal extremities of femur), tibia (normal ossification center at proximal extremity of tibia) were detected (Plates 3B.3C and 3D).

The examination of the fifth group, in the fore appendicular skeleton, future bony structures of scapula, humerus (normal
Plate 1 (C): A radiographic image of rabbit skeleton (first control group) (bilateral view) showing, skull and mandible (a), humerus (c), ulna (d), ribs (f), vertebral column (g), tibia (j) and caudal vertebrae (tail) (k), normal ossification center at proximal extremity of humerus (1), normal ossification center at distal extremity of humerus (2) and normal ossification center at proximal extremity of tibia (5).

Plate 1 (D): A radiographic image of rabbit skeleton (second group) (dorsoventral view) showing, skull (a), scapula (b), humerus (c), ulna (d), radius (e), ribs (f), vertebral column (g), coxal bone (h), femur (i), tibia (j) and abnormal absence of last six caudal vertebrae (tail) (k), abnormal absence of ossification center at proximal extremity of humerus (1), normal ossification center at distal extremity of humerus (2), abnormal absence of ossification center at proximal extremity of femur (3), abnormal absence of ossification center at distal extremity of femur (4) and normal ossification center at proximal extremity of tibia (5).

ossification center at proximal extremity of humerus, normal ossification center at distal extremity of humerus), radius (normal ossification center at distal extremity of radius), ulna (normal ossification center at distal extremity of ulna) were clarified. In the hind appendicular skeleton, future bony structures of coxal bone, femur (normal ossification center at proximal extremity of femur, normal ossification center at distal extremity of femur), tibia (normal ossification center at proximal extremity of tibia) were detected (Plate. 4A,4B and $4 \mathrm{C})$. At the radiographic image of rabbit skeleton of the fourth and fifth groups, abnormal curved vertebral column was observed (Plates 3C, 3D, 4A and 4B). 

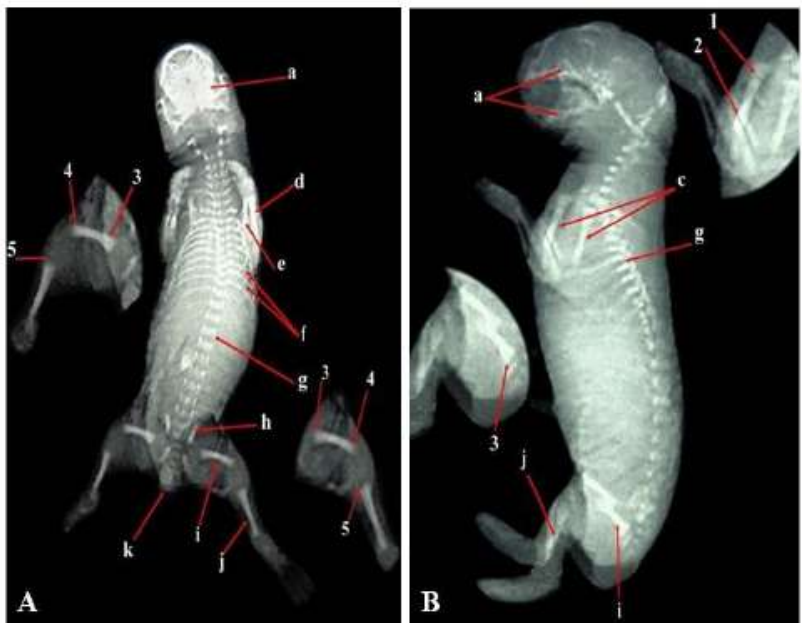

\section{Discussion}

The present investigation revealed that, radiographic examination of the fore limb of the foeti in the control group showed normal secondary ossification centers at proximal and distal extremities of humerus, these results were in agreement with a previous study [2]. In dogs, there was one secondary ossification center only for diaphysis appeared at full term [19]. Moreover, in a pig fetus, four centers were
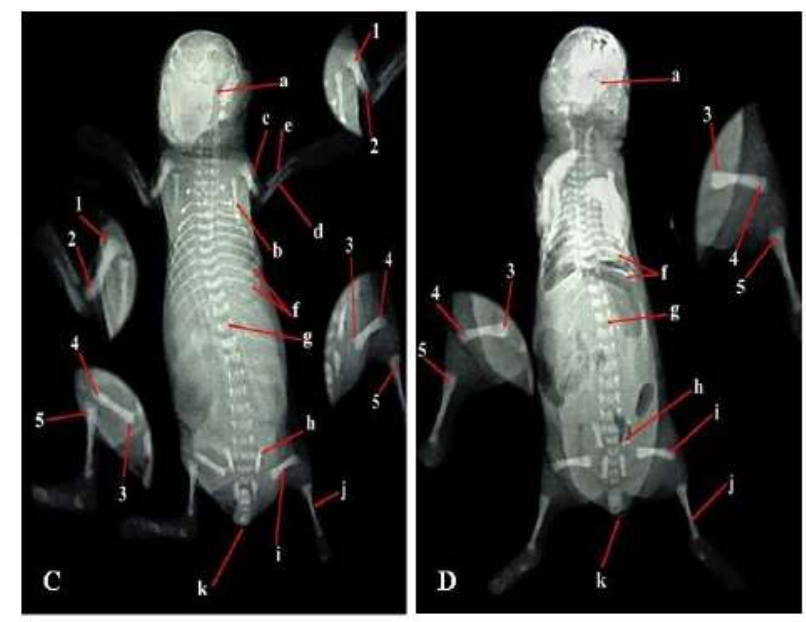

Regarding the examination of the hind limb of the control group, normal secondary ossification center at proximal extremity of tibia was observed. This was in agreement with other studies reported in rabbits [2]. The appearance of secondary ossification center at proximal epiphysis of tibia did not occur in dog fetus, which is contrary to the rabbit tibia [21,22]. While in pig fetus, another third secondary ossification center appeared at the
Plate 2 (A): A radiographic image of rabbit skeleton (second group) (ventrodorsal view) showing, skull (a), ulna (d), radius (e), ribs (f), vertebral column (g), coxal bone (h), femur (i), tibia (j) and caudal vertebrae (tail) (k), abnormal absence of ossification center at proximal extremity of femur (3), abnormal absence of ossification center at distal extremity of femur (4) and normal ossification center at proximal extremity of tibia (5).

Plate 2 (B): A radiographic image of rabbit skeleton (second group) (bilateral view) showing, skull and mandible (a), humerus (c), vertebral column (g), femur (i) and caudal vertebrae (tail) (k), abnormal absence of ossification center at proximal extremity of humerus (1), normal ossification center at distal extremity of humerus (2) and abnormal absence of ossification center at proximal extremity of femur (3).

detected as one for diaphysis, bilateral and medial tuberosities and head of humerus [20].

During the radiographic examination of the hind limb of the control group, two ossification centers were found for the femur (proximal and distal extremities). These results were in agreement with other findings in rabbits [2]. In contrary, only one center was present at full term of pregnancy in rats [21] and dogs [2224], while, four centers were observed in pig fetus [20].

Plate 2 (C): A radiographic image of rabbit skeleton (third group) (dorsoventral view) showing, skull (a), scapula (b), humerus (c), radius (d), ulna (e), ribs (f), vertebral column (g), coxal bone (h), femur (i), tibia (j) and absence of last four caudal vertebrae (tail) (k), abnormal absence of ossification center at proximal extremity of humerus (1), normal ossification center at distal extremity of humerus (2), abnormal absence of ossification center at proximal extremity of femur (3), normal ossification center at distal extremity of femur (4) and abnormal absence of ossification center at proximal extremity of tibia $(5)$.

Plate 2 (D): A radiographic image of rabbit skeleton (third group) (ventrodorsal view) showing, skull (a), ribs (f), vertebral column (g), coxal bone $(h)$, femur (i), tibia (j) and caudal vertebrae (tail) (k), abnormal absence of ossification center at proximal extremity of femur (3), normal ossification center at distal extremity of femur (4) and abnormal absence of ossification center at proximal extremity of tibia (5).

distal epiphysis of tibia, such finding is not reported in rabbits [20].

This study provided a better knowledge about the critical developmental time for different segments of the rabbit skeleton to investigate malformations. During the X-ray observations of the foeti from the second group of which pregnant rabbits were injected with excess dose of vitamin $\mathrm{A}$ at $3^{\text {rd }}-6^{\text {th }}$ gestation day and those from the third group injected at 
$7^{\text {th }}-10^{\text {th }}$ gestation day, there were some anatomical changes at axial skeleton. In accordance, the axial skeletal development in rabbits was determined in pregnancy during $8^{\text {th }}$ to $13^{\text {th }}$ gestation days [25].
The secondary ossification centers at definite proximal and distal extremities of long bones were formed prenatally and were examined in full term foeti by X-ray. This was inconsistent with the other findings where the maturation process from fusion of the secondary ossification centers of long bone extremities was postnatally in Wistar rats [26].
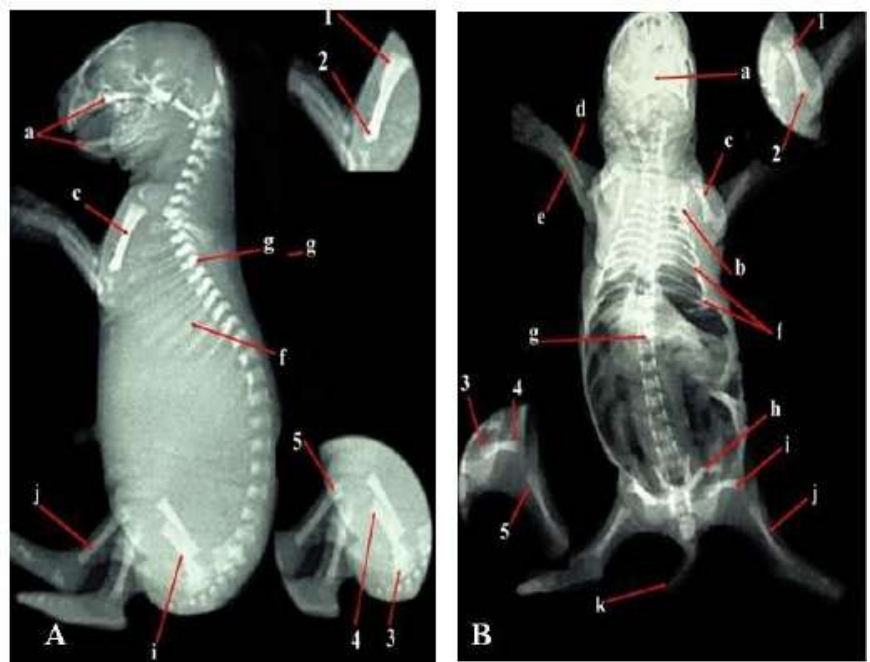

Plate 3 (A): A radiographic image of rabbit skeleton (third group) (bilateral view) showing, skull and mandible (a), humerus (c), ribs (f), vertebral column (g), femur (i) and tibia (j), abnormal absence of ossification center at proximal extremity of humerus (1), abnormal absence of ossification center at distal extremity of humerus (2), normal ossification center at proximal extremity of femur (3), abnormal absence of ossification center at distal extremity of femur (4) and abnormal absence of ossification center at proximal extremity of tibia (5).

Plate 3 (B): A radiographic image of rabbit skeleton (fourth group) (dorsoventral view) showing, skull (a), scapula (b), humerus (c), radius (d), ulna (e), ribs (f), vertebral column $(\mathrm{g})$, coxal bone $(\mathrm{h})$, femur $(\mathbf{i})$, tibia $(\mathbf{j})$ and caudal vertebrae (tail) (k), normal ossification center at proximal extremity of humerus (1), abnormal absence of ossification center at distal extremity of humerus (2), abnormal absence of ossification center at proximal extremity of femur (3), normal ossification center at distal extremity of femur (4) and normal ossification center at proximal extremity of tibia (5).

The radiographic examination of the fore and hind limbs of the control group showed normal ossification centers at proximal and distal extremities of humerus, proximal and distal extremities of femur and at proximal extremity of tibia. In accordance, other findings confirmed that radiographically, evidence of closure of the growth plates in the distal femur occurred at 20-23 weeks and in the proximal tibia at 22-27 weeks postnatally [27].
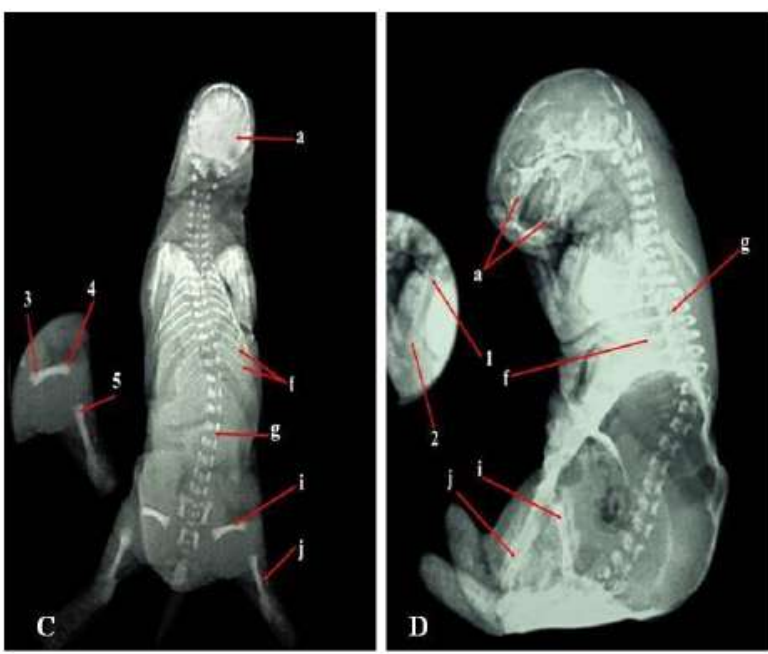

The secondary ossification centers which were detected by X-ray examination of the fore and hind limbs in the second group were in agreement with other observations in rabbits [2]. The abnormal absence of the secondary ossification centers at the proximal extremities of the humerus and femur; distal extremity of the femur was in contrary with other studies in rabbits [2], rats [26] and monkeys [28].

Plate $3(\mathrm{C})$ : A radiographic image of rabbit skeleton (fourth group) (ventrodorsal view) showing, skull (a), ribs (f), vertebral column (g), coxal bone (h), femur (i) and tibia (j), abnorma absence of ossification center at proximal extremity of femur (3), abnormal absence of ossification center at distal extremity of femur (4) and abnormal absence of ossification center at proximal extremity of tibia (5).

Plate 3 (D): A radiographic image of rabbit skeleton (fourth group) (bilateral view) showing, skull and mandible (a), ribs (f), abnormal curved vertebral column (g), femur (i) and tibia (j), abnormal absence of ossification center at proximal extremity of humerus (1) and abnormal absence of ossification center at distal extremity of humerus (2). 
The present study pointed out that, the Xray examination of the fore and hind limbs of the third, fourth and fifth groups revealed abnormal absence of ossification centers at proximal and distal extremities of humerus and femur and proximal extremity of tibia. These results indicate the effect of hypervitaminosis $A$ on the formation of these centers of ossification at the critical developmental time. This opinion was confirmed by Abdelaziz et al. [2] who stated that these centers were visualized at late stage of pregnancy (at three days).
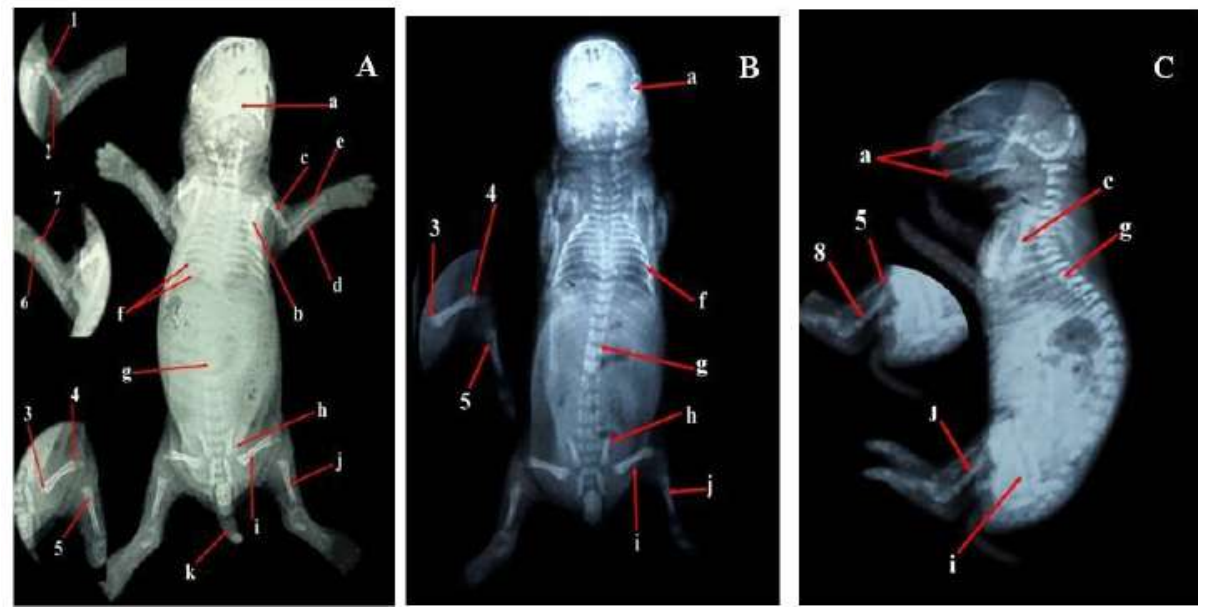

Plate 4 (A): A radiographic image of rabbit skeleton (fifth group) (dorsoventral view) showing, skull (a), scapula (b), humerus (c), ulna (d), radius (e), ribs (f), vertebral column (g), coxal bone (h), femur (i), tibia (j) and caudal vertebrae (tail) (k), normal ossification center at proximal extremity of humerus (1), normal ossification center at distal extremity of humerus (2), normal ossification center at proximal extremity of femur (3), normal ossification center at distal extremity of femur (4), normal ossification center at proximal extremity of tibia (5), abnormal ossification center at distal extremity of radius (6) and abnormal ossification center at distal extremity of ulna (7). (B): A radiographic image of rabbit skeleton (fifth group) (ventrodorsal view) showing, skull (a), ribs (f), vertebral column (g), coxal bone (h), femur (i) and tibia (j), abnormal absence of ossification center at proximal extremity of femur (3), normal ossification center at distal extremity of femur (4) and normal ossification center at proximal extremity of tibia (5). (C): A radiographic image of rabbit skeleton (fifth group) (bilateral view) showing, skull and mandible (a), humerus (c), vertebral column (g), femur (i) and tibia (j), normal ossification center at proximal extremity of humerus (1), normal ossification center at distal extremity of humerus (2), normal ossification center at proximal extremity of tibia (5) and abnormal ossification center at distal extremity of tibia (8).

\section{Conclusion}

In conclusion, the injection of rabbit does by vitamin A must be with caution during pregnancy because it might cause hypervitaminosis A leading to several skeletal malformations in offspring especially on ossification centers particularly from $3^{\text {rd }}$ to $19^{\text {th }}$ days of gestation which is considered a critical stage.

\section{Conflict of interest}

The authors declare no conflict of interest.

\section{References}

[1] Lippman, S.M.; Benner, S.E. and Hong, W.K. (1993): Chemoprevention. Strategies for the control of cancer. Cancer, 72 (3 suppl): 984-990.
[2] Abdelaziz, S.E.; Nosseur, H.M. and Selim, A.A. (1992): Observation on the ossification centers in rabbit fetus. Egyptian J Rabbit Science, 2 (2): 171-183.

[3] Winkelmann, C.T. and Wise, L.D. (2009): High-through put micro-computed tomography imaging as a method to evaluate rat and rabbit fetal skeletal abnormalities for developmental toxicity studies. J Pharmacol Toxicol Methods, 59:156-165.

[4] Jorgensen, S.M.; Demirkaya, O. and Ritman, E.L. (1998): Three-dimensional imaging of vasculature and parenchyma in intact rodent organs with X-ray micro-CT. Am J Physiol, 275 (3 Pt 2): 1103-1114.

[5] Paulus, M.J.; Gleason, S.S.; Kennel, S.J.; Hunsicker, P.R., and Johnson, D.K. (2000): 
High resolution X-ray computed tomography: An emerging tool for small animal cancer research. Neoplasia, 2 (1-2): 62-70.

[6] Ritman, E.L. (2004). Micro-computed tomography current status and developments. Annu Rev Biomed Eng, 6: 185-208.

[7] French, J.; Halliday, J.; Scott, M.; Adkins, D.; Liess, C.; Waterton, J.C. and Stewart, J. (2010): Retinal folding in the term rabbit fetus developmental abnormality or fixation artifact. Reprod Toxicol, 26(34):262-266.

[8] Kimmel, C.A. and Price, C.J. (1990): Developmental toxicity studies. In: Handbook of in vivo Toxicity Testing. Arnold D.L.; Grice, H.C. and Krewski, D.R. (Eds.). San Diego, CA: AP, pp. 271299

[9] Kimmel, C.A. and Trammel, C. (1981): A rapid procedure for routine double staining of cartilage and bone in fetal and adult animals. Stain Technol, 56 (5):271-273.

[10] Nothdurft, H.; Sterz, H. (1977): Routine radiography of the skeletons of 31-day-old rabbit feotei. In: Methods prenatal toxicology [Teratol Workshop]. pp. 155164.

[11]Guldberg, R.E.; Lin, A.S.; Coleman, R.; Robertson, G. and Duvall, C. (2004): Microcomputed tomography imaging of skeletal development and growth. Birth Defects Res C Embryo Today, 72 (3):250259.

[12] Wise, L.D. and Winkelmann, C.T. (2009): Micro-computed tomography and Alizarin red evaluations of boric acid-induced fetal skeletal changes in Sprague-Dawley Rats. Birth Defects Res B Dev Reprod Toxicol, 86(3): 214-219

[13] Fukuda, S. and Matsuoka, O. (1979): Maturation process of secondary ossification centers in the rat and assessment of bone age. Jikken Dobutsu, 28(1):1-9.
[14] Radostits, O.M.; Gay, C.C.; Hinchcliff, K.W. and Constable, P.B. (2007): Veterinary Medicine, $10^{\text {th }}$ ed. Saunders, Edinburgh. pp. 1771-1777.

[15] Hough, S.; Avioli, L.V.; Muir, H.; Gelderblom, D.; Jenkins, G.; Kurasi, H.; Slatopolsky, E.; Bergfeld, M.A. and Teitelbaum, S.L. (1988): Effects of hypervitaminosis $\mathrm{A}$ on the bone and mineral metabolism of the rat. Endocrinology, 122(6): 2933-2939.

[16] Kamm, J.J.; Ashenfelter, K.O. and Ehmann, C.W. (1984): Preclinical and clinical toxicology of selected retinoids. In: Sporn, M.B.; Roberts, A.B., and Goodman, D.S., (Eds.). The Retinoids. Orlando, Academic Press,Inc., pp. 287-326.

[17] Zbinden, G. (1975): Pharmacology of vitamin A acid (beta-all-transretinoic acid). Acta Dermatol Venerol, (55 Suppl.), 74:21-24.

[18] Yochum, T.R.; Rowe, L.J. and Lindsay J. (1996): Essentials of skeletal radiology. $2^{\text {nd }}$ Ed. Williams \& Wilkins, United States.

[19] Hare, W.C.D. (1959): Radiographic anatomy of the canine pectoral limb. Part. II: Developing limb. J Am Vet Med Assoc, 136: 305-310.

[20] Wenham, G.; Mc Donald, I. and Elsley, F.W.H. (1969): Radiographic study of the development of the skeleton of fetal pig. $\mathrm{J}$ Agric Sci, 72 (1): 123-130.

[21] Strong, R.M. (1925): The order, time and rate of ossification of albino rat skeleton. Am J Anat, 36: 313-320.

[22] Chapman, W.L. (1965): Appearance of ossification centre and epiphysial closures as determined by radiographic techniques. J Am Vet Med Assoc, 147 (2): 138-144.

[23] Hare, W.C. (1960): Radiographic anatomy of the canine pelvic limb. Part. II: Developing limb. J Am Vet Med Assoc, 136: 603-611. 
[24] Anderson, A.C. and Floyd, M. (1963): Growth and development of the femur in the beagle. Am J Vet Res, 24: 348-351.

[25] Campion, S.N.; Davenport, S.J.; Nowland, W.S.; Cappon, G.D.; Bowman, C.J. and Hurtt, M.E. (2012): Sensitive windows of skeletal development in Rabbits determined by hydroxyurea exposure at different times throughout gestation. Birth Defects Res B Dev Reprod Toxicol, 95 (3):238-249.

[26]Gentili, P.; Trasimeni, A. and Giorlandino, C. (1984): Fetal ossification centers as predictors of gestational age in normal and abnormal pregnancies. J Ultrasound Med, 3(5):193-197.
[27] Kaweblum, M.; Aquilar, M.C.; Blancas, E.; Kaweblum, J.; Lehman, W.B.; Grant, A.D. and Strongwater, A.M. (1994): Histological and radiographic determinatio $n$ of the age of physeal closure of the distal femur, proximal tibia, and proximal fibula of the New Zealand white rabbit. J Orthop Res, 12(5):747-749.

[28] Fukuda, S.; Cho, F. and Honjo, S. (1978): Bone growth and development of secondary ossification centers of extremities in the cynomolgus monkey (Macaca fascicularis). Jikken Dobutsu, 27(4):387-397.

$$
\begin{aligned}
& \text { الملخص العربي } \\
& \text { دراسات بالاشعة السينية علي أجنة الارانب تحت تأثير الجرعة الزائدة من فيتامين أ }
\end{aligned}
$$

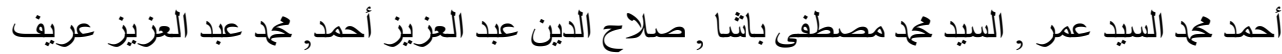

$$
\begin{aligned}
& \text { قسم التشريح و الاجنةـ كلية الطب البيطرى- جامعة الزقازيق النقاين }
\end{aligned}
$$

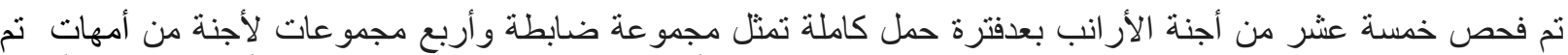

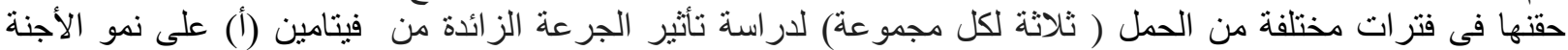

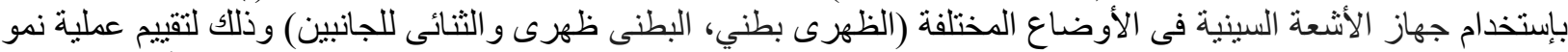

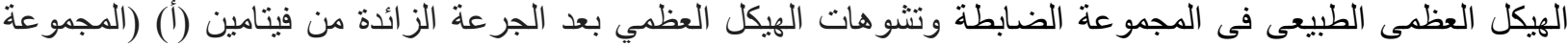

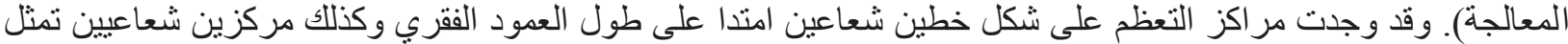

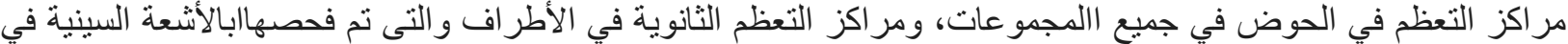

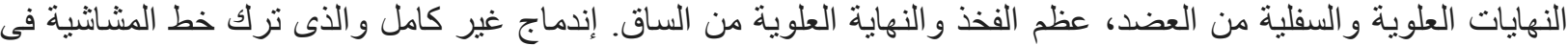

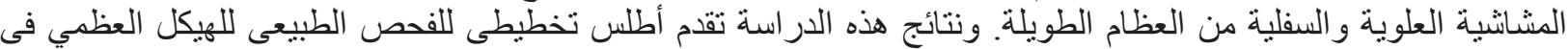

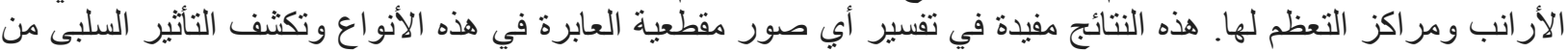
فرط استخدام فيتامين (أ) أثناء فترات التهمل العن على تطوير الهيكل العظمي لأجنتها. 